\title{
A Manifold Learning Approach to Data-Driven Computational Materials and Processes
}

\author{
Ruben Ibañez ${ }^{1, b)}$, Emmanuelle Abisset-Chavanne ${ }^{1, \mathrm{c})}$, Jose Vicente Aguado ${ }^{1, \mathrm{~d})}$, \\ David Gonzalez $^{2, \mathrm{e})}$, Elias Cueto ${ }^{2, \mathrm{a}), \mathrm{f})}$, Jean Louis Duval ${ }^{3, \mathrm{~g})}$ and Francisco \\ Chinesta $^{1, \mathrm{~h})}$

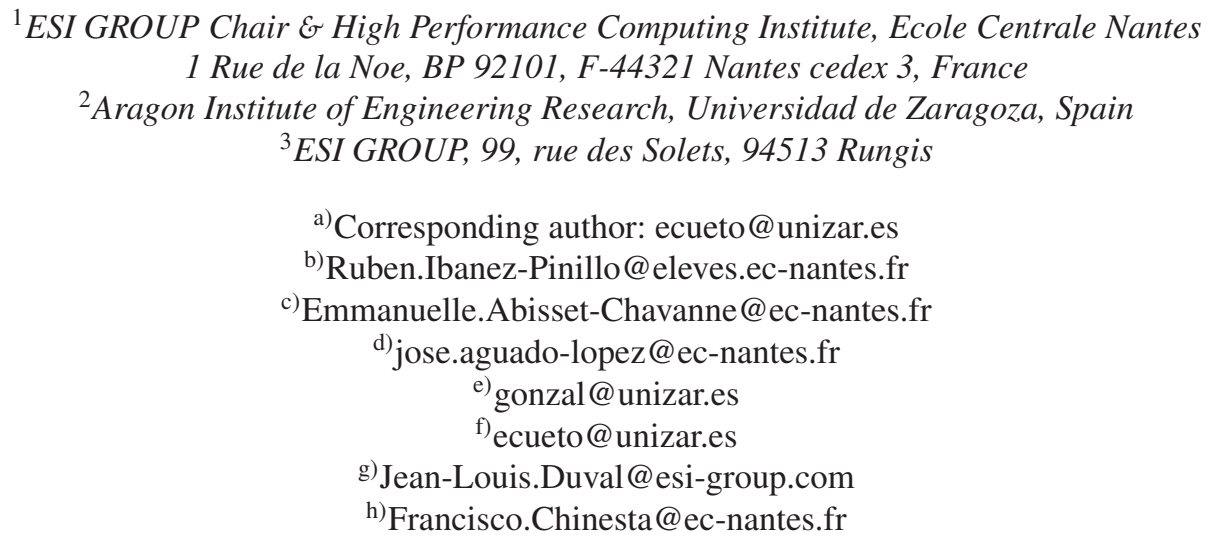

\begin{abstract}
Standard simulation in classical mechanics is based on the use of two very different types of equations. The first one, of axiomatic character, is related to balance laws (momentum, mass, energy, ...), whereas the second one consists of models that scientists have extracted from collected, natural or synthetic data. In this work we propose a new method, able to directly link data to computers in order to perform numerical simulations. These simulations will employ universal laws while minimizing the need of explicit, often phenomenological, models. They are based on manifold learning methodologies.
\end{abstract}

\section{INTRODUCTION}

In the present work we will assume that all the needed data is available. We will not address all the difficulties related to data generation or obtention from adequate experiments. This is a topic that, of course, remains open. On the contrary, we develop a method in which this stream of data plays the role of a constitutive equation, without the need of a phenomenological fitting to a prescribed model.

To better understand the data-driven rationale addressed in the present work, let us consider, for the sake of clarity, a very simple problem: linear elasticity. In that case the balance of (linear and angular) momentum leads to the existence of a symmetric second-order tensor $\sigma$ (the so-called Cauchy's stress tensor) verifying equilibrium, expressed in the absence of body forces, as:

Find the displacement field $\mathbf{u} \in\left(\mathcal{H}^{1}(\Omega)\right)^{3}$ satisfying the essential boundary conditions $\mathbf{u}\left(\mathbf{x} \in \Gamma_{D}\right)=\mathbf{u}_{g}(\mathbf{x})$ such that

$$
\int_{\Omega} \varepsilon^{*}: \sigma d \mathbf{x}=\int_{\Gamma_{N}} \mathbf{u}^{*} \cdot \mathbf{t} d \mathbf{x}
$$

$\forall \mathbf{u}^{*}$ regular enough and vanishing on $\Gamma_{D}$, i.e. $\forall \mathbf{u}^{*} \in\left(\mathcal{H}_{0}^{1}(\Omega)\right)^{3}$. 
The weak form given by Eq. (1) involves kinematic and dynamic variables from the test displacement field $\mathbf{u}^{*}$ and the stress tensor $\sigma$ respectively. In order to solve it a relationship linking kinematic and dynamic variables is required, the so-called constitutive equation. The simplest one, giving rise to linear elasticity, is known as Hooke's law (even if, more than a law, it is simply a model), and writes

$$
\boldsymbol{\sigma}=\lambda \operatorname{Tr}(\boldsymbol{\varepsilon}) \mathbf{I}+\mu \boldsymbol{\varepsilon},
$$

where $\operatorname{Tr}(\bullet)$ denotes the trace operator, and $\lambda$ and $\mu$ are the Lame coefficients directly related to the Young modulus $E$ and the Poisson coefficient $v$.

By introducing the constitutive model, Eq. (2), into the weak form of the balance of momentum, Eq. (1), a problem is obtained that can be formulated entirely in terms of the displacement field $\mathbf{u}$. By discretizing it, using standard finite element approximations, for instance, and performing numerically the integrals involved in Eq. (1), we finally obtain a linear algebraic system of equations, from which the nodal displacements can be obtained.

In the case of linear elasticity there is no room for discussion: the approach is simple, efficient and has been applied successfully to many problems of interest. Today, there are numerous commercial codes making use of this mechanical behavior and nobody doubts about its pertinence in engineering practice. However, there are other material behaviors for whom simple models fail to describe any experimental finding. These models lack of generality (universality) and for this reason a mechanical system is usually associated to different models that are progressively adapted and/or enriched from the collected data.

The biggest challenge could then be formulated as follows: can simulation proceed directly from data by circumventing the necessity of establishing a constitutive model? In the case of linear elasticity it is obvious that such an approach lacks of interest. However, in other branches of engineering science and technology it should be an appealing alternative to standard constitutive model-based simulations. In our opinion, we are at the beginning of a new era, the one of data-based or, more properly, data-driven engineering science and technology, where as much as possible data should be collected and information extracted in a systematic way by using adequate machine learning strategies. Then, simulations could proceed directly from this automatically acquired knowledge.

\section{CONSTITUTIVE MANIFOLD}

We consider first mechanical tests conducted on a perfectly linear elastic material, in a specimen exhibiting uniform stresses and strains. As previously indicated, in this work we do not address issues related to data generation. Thus, for $M$ randomly applied external loads, we assume ourselves able to collect $M$ couples $\left(\boldsymbol{\sigma}_{m}, \boldsymbol{\varepsilon}_{m}\right), m=1, \ldots, M$. These pairs could be represented as a single point $\mathbf{X}_{m}$ in a phase space of dimension $D=12$ (the six distinct components of the stress and strain tensors, respectively). In the sequel Voigt notion will be considered, i.e. stress and strain tensors will be represented as vectors and the fourth-order elastic tensor reduces to a square matrix.

Each vector $\mathbf{X}_{m}$ thus defines a point in a space of dimension $D$ and, therefore, the whole set of samples represents a set of $M$ points in $\mathbb{R}^{D}$. We conjecture that all these points belong to a certain low-dimensional manifold embedded in the high-dimensional space $\mathbb{R}^{D}$ allowing for a nonlinear dimensionality reduction as discussed in [2]. In what follows we proceeds without such a dimensionality reduction and consider the simplest strategy proposed and discussed in [2]. We consider locally linear approximations, that allow writing

$$
\mathbf{X}_{m}=\sum_{i=1}^{M} W_{m i} \mathbf{X}_{i},
$$

with $W_{m i}=0$ if $i \notin \mathcal{S}_{m}$ (set containing the $K$-nearest neighbors of $\mathbf{X}_{m}$ ). By minimizing the functional

$$
\mathcal{H}(\mathrm{C})=\sum_{i \in \mathcal{S}_{m}}\left(\sigma_{i}-\mathrm{C} \cdot \boldsymbol{\varepsilon}_{i}\right)^{2} .
$$

we obtain $\mathrm{C}\left(\mathbf{X}_{m}\right) \equiv \mathrm{C}_{m}$. 


\section{DATA-DRIVEN SIMULATION}

From the just identified locally linear behavior $\mathbf{C}(\mathbf{X})$ one could apply the simplest explicit linearization technique operating on the standard weak form

$$
\int_{\Omega} \varepsilon^{*}(\mathbf{x}): \sigma^{n+1}(\mathbf{x}) d \mathbf{x}=\int_{\Gamma_{N}} \mathbf{u}^{*}(\mathbf{x}) \cdot \mathbf{t}(\mathbf{x}) d \mathbf{x}
$$

where at each point, from the stress-strain couple at position $\mathbf{x}, \mathbf{X}(\mathbf{x})$, the locally linear behavior $\mathbf{C}(\mathbf{X}(\mathbf{x}))$ can be obtained (in practice at the Gauss points used for the integration of the weak form) that allows us to write (using Voigt notation)

$$
\int_{\Omega} \boldsymbol{\varepsilon}^{*}(\mathbf{x}) \cdot(\mathrm{C}(\mathbf{x}) \cdot \boldsymbol{\varepsilon}(\mathbf{x})) d \mathbf{x}=\int_{\Gamma_{N}} \mathbf{u}^{*}(\mathbf{x}) \cdot \mathbf{t}(\mathbf{x}) d \mathbf{x} .
$$

This allows, in turn, to compute the displacement field and from it, to update the strain and stress fields, to compute again the locally linear behavior. The process continues until convergence. The discretization related to the other two descriptions just introduced were deeply considered in [2].

\section{NUMERICAL RESULTS}

\section{A simple nonlinear behavior: the linear-elastic perfectly-plastic behavior}

We start by addressing the case of a linear-elastic-perfectly plastic 2D behavior. We assume the linear elastic contribution defined locally from $\mathbf{C}\left(\mathbf{X}^{e}\right)$ ( $\mathbf{X}^{e}$ refers to the stress-elastic strain manifold) whereas the plastic contribution that involves the yield surface $f(\boldsymbol{\sigma})$ is assumed given by its own manifold.

Using again Voigt notation, the elastic behavior expressed from $\sigma=\mathrm{C} \cdot \boldsymbol{\varepsilon}^{e}$, where $\mathrm{C}$ represents the manifoldbased elastic tensor and $\varepsilon^{e}$ refers to the elastic component of the deformation (the reversible one). The total strain can be decomposed in its elastic and inelastic components,

$$
\varepsilon=\varepsilon^{e}+\varepsilon^{p}
$$

where we assume the plastic flow rate

$$
\dot{\boldsymbol{\varepsilon}}^{p}=\lambda \frac{\partial f(\sigma)}{\partial \sigma}=\lambda \mathbf{n},
$$

where the yield surface $f(\sigma)$ is provided by experimental data. To generate these data in silico, we assume that it follows a von Mises model $f(\sigma)=\sigma_{e}-Y$, with $Y$ the yield stress (no hardening is considered) and $\sigma_{e}$ the equivalent stress related to the von Mises criterion. $f(\sigma)$ results in the surface represented in blue in Fig. 1 where, for the sake of clarity, it is represented in the space of stresses.

The persistency condition $\dot{f}(\sigma)=0$ when plastic flow occurs, results in the following plastic flow

$$
\lambda=\frac{\mathbf{n}^{T} \cdot \mathrm{C} \cdot \dot{\boldsymbol{\varepsilon}}}{\mathbf{n}^{T} \cdot \mathrm{C} \cdot \mathbf{n}}
$$

or in its incremental counterpart

$$
\lambda=\frac{\mathbf{n}^{T} \cdot \mathrm{C} \cdot \Delta \boldsymbol{\varepsilon}}{\mathbf{n}^{T} \cdot \mathrm{C} \cdot \mathbf{n}}
$$

with now $\Delta \boldsymbol{\varepsilon}^{p}=\lambda \mathbf{n}$.

Here three fields must be considered, stress, strain and plastic strain. As soon as the last one is known, the elastic strain can be locally determined and the stresses obtained from the elastic manifold using the couple stress-elastic component of the strain.

In these expressions everything is properly defined except $\mathbf{n}$, since we assume that the explicit form of the yield condition, i.e. $f(\sigma)$ is unknown and the only available data is the manifold depicted in Fig. 1. However, $\mathbf{n}$ is easily accessible by considering the normal vector to the plastic manifold (blue cylinder depicted in Fig. 1). 


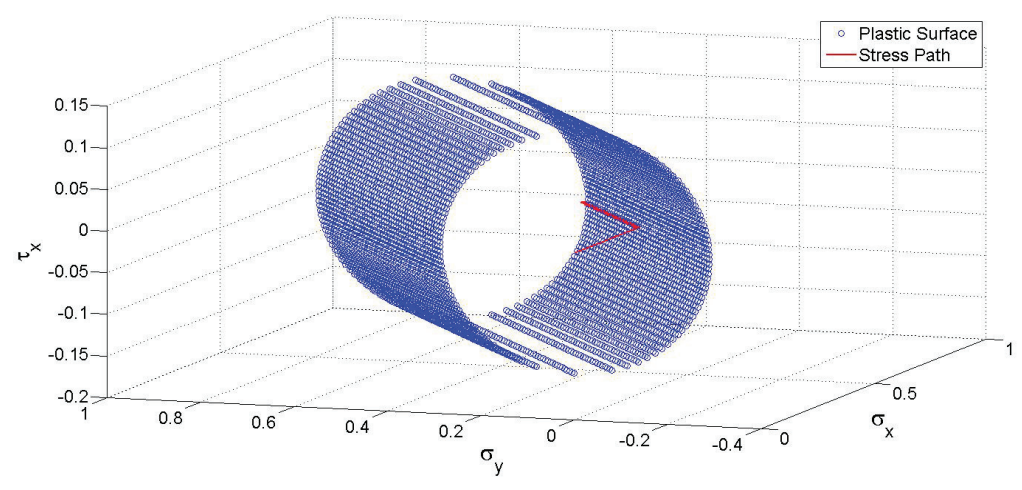

FIGURE 1. Stress trajectory in the stress space in the elastic-perfecly plastic behavior
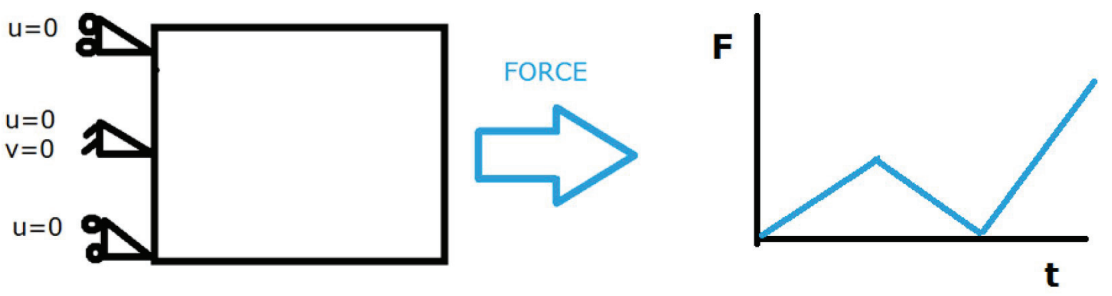

FIGURE 2. Mechanical problem and loading trajectory

Now one could imagine performing a standard linear elastic-perfectly plastic simulation by using a finite element explicit code where the plastic deformation is computed from the manifold that allows extracting $\mathbf{n}$ instead of the knowledge of function $f(\sigma)$ and its explicit derivative with respect to the stresses.

When considering the traction of a square domain along its right side, with appropriate boundary conditions on its left side (with tension-free conditions on the top and bottom boundaries) ensuring an homogeneous stress and strain fields everywhere in the domain, the stress trajectory in the stress space is depicted in Fig. 1. It can be noticed that the elastic behavior applies when the stress remains inside the plastic surface and then it remains in the surface during the plastic flow. Again, for the sake of simplicity, the results are shown in the stress domain.

\section{Isotropic hardening}

Isotropic hardening can be easily addressed from its own manifold relating hardening with plastic deformation for example, and the preceding as usually. More complex and general alternatives exist. based on the only use of data. When considering again the test depicted in Fig. 2 the evolution of the yield surface due to the hardening is depicted in Fig. 3.

\section{Anisotropic yield function}

We could also consider more complex data, as the one associated to anisotropic yields widely considered by Barlat and coauthors [1,4]. By restricting to plane stress data one could obtain the plastic surface represented in Fig. 4 (blue points). The superposed curve represents the stress history at a certain Gauss point with the color representing the loading parameters. The elastic and elastoplastic regimes can be appreciated. Even if in this case the represented data can be convenablely approximated by a standard anisotropic yield, the procedure here described could avoid its explicit use. 

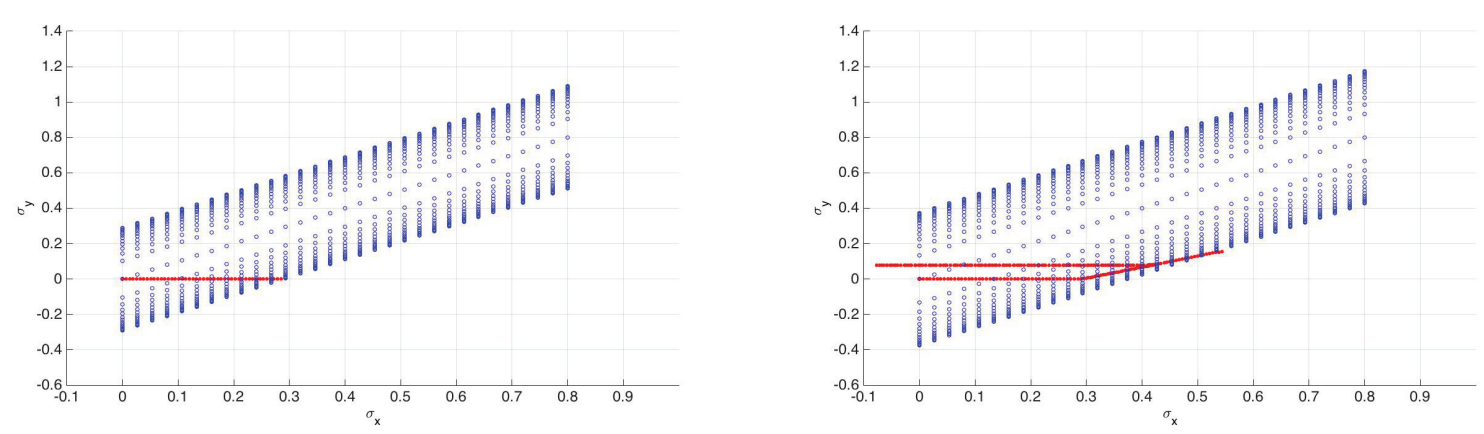

FIGURE 3. Yield surface evolution due to the material hardening

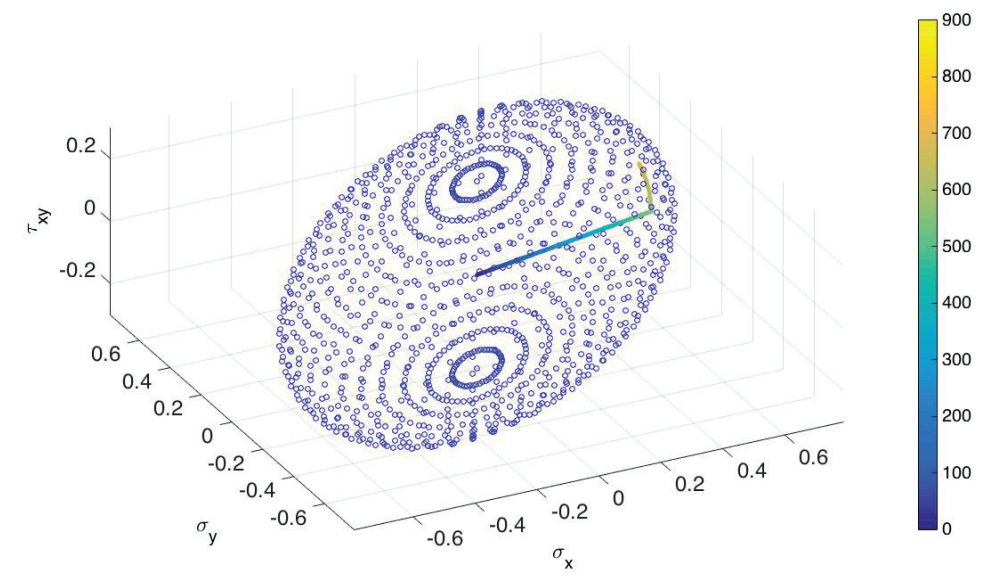

FIGURE 4. Loading trajectory when considering an anisotropic plastic yield

\section{CONCLUSION}

This work illustrates how to alleviate the use of constitutive models with respect the simple consideration of data coming from appropriate experimental tests. The issue related to such tests is not discussed here, however it is important to note that that issue can be successfully circumvented by considering complex tests and using appropriate manifold-based inverse analyses. This kind of analysis will be reported in future works.

\section{REFERENCES}

[1] F. Barlat, H. Aretz, J.W. Yoon, M.E. Karabin, J.C. Brem, R.E. Dick. Linear transfomation-based anisotropic yield functions. International Journal of Plasticity, 21, 1009-1039, 2005.

[2] R. Ibanez, E. Abisset-Chavanne, J.V. Aguado, D. Gonzalez, E. Cueto, F.Chinesta. A manifold learning approach to data-driven computational elasticity and inelasticity, Archives of Computational Methods in Engineering, DOI 10.1007/s11831-016-9197-9

[3] S.T. Roweis, L.K. Saul. Nonlinear dimensionality reduction by locally linear embedding, Science, 290/5500, 2323-2326, 2000.

[4] J.W. Yoon, F. Barlat, R.E. Dick, M.E. Karabin. Prediction of six or eight ears in a drawn cup based on a new anisotropic yield function. International Journal of Plasticity 22, 174-193, 2006. 